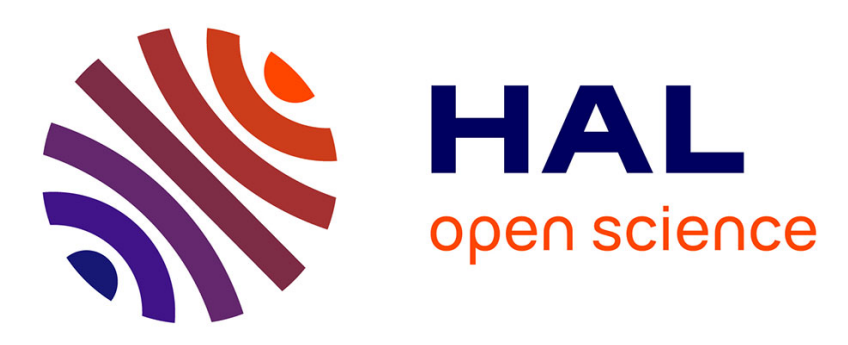

\title{
Active fault tolerant control design for switched hybrid systems
}

Mickael Rodrigues, Didier Theilliol, Dominique Sauter

\section{To cite this version:}

Mickael Rodrigues, Didier Theilliol, Dominique Sauter. Active fault tolerant control design for switched hybrid systems. 2nd IFAC Symposium on Analysis and Design of Hybrid Systems, ADHS'06, Jun 2006, Alghero Sardinia, Italy. pp.CDROM. hal-00095581

\section{HAL Id: hal-00095581 https://hal.science/hal-00095581}

Submitted on 2 Mar 2009

HAL is a multi-disciplinary open access archive for the deposit and dissemination of scientific research documents, whether they are published or not. The documents may come from teaching and research institutions in France or abroad, or from public or private research centers.
L'archive ouverte pluridisciplinaire HAL, est destinée au dépôt et à la diffusion de documents scientifiques de niveau recherche, publiés ou non, émanant des établissements d'enseignement et de recherche français ou étrangers, des laboratoires publics ou privés. 


\title{
FAULT TOLERANT CONTROL DESIGN FOR SWITCHED SYSTEMS
}

\section{Mickaël Rodrigues, Didier Theilliol and Dominique Sauter}

\author{
Centre de Recherche en Automatique de Nancy, \\ CRAN-UMR-CNRS 7039 Université Henri Poincaré \\ Nancy I, B.P. 239, 54506 Vandoeuvre-lès-Nancy Cedex, \\ France. Phone: +33 $383684480-$ Fax: + 33383684462 \\ E-mail: mickael.rodrigues@cran.uhp-nancy.fr
}

\begin{abstract}
In this paper, we develop a Fault Tolerant Control (FTC) strategy designed to preserve closed-loop stability in spite of actuator failures. The main contribution is the design of a linear output feedback control function for a class of discrete-time switched linear systems with explicit modelling of multiple actuator failures. Assuming an on-line and real-time Fault Detection and Isolation (FDI) scheme such that, each time there is a change in status of one or more of the $p$ many actuators, the FDI scheme will update a p-length vector of real parameters in the interval $[0,1]$ recording the degree of loss of effectiveness (with 0 for normal operation and 1 for total failure), we can perform controller redesign on-line using LMI techniques. We conclude the paper with an example illustrating the performance of our proposed FTC strategy. Copyright (c) 2006 IFAC
\end{abstract}

Keywords: Fault Tolerant Control, Switched Systems, Actuator Failure, LMI, Output Feedback, Stability

\section{INTRODUCTION}

In recent years, the study of switched systems has received a growing attention in control theory and practice. By switched systems we mean a class of hybrid dynamical systems consisting of a family of continuous (or discrete) time subsystems and a rule that governs the switching between them. A survey of basic problems in stability and design of switched systems is given in (Liberzon and Morse, 1999) where some contributions are summarized. Most of these contributions deal with stability analysis or design of state feedback based control laws in fault-free case (Mignone et al., 2000), (Daafouz and Bernussou, 2002), (Chadli et al., 2002), (Daafouz et al., 2003) but rarely in faulty case. This paper aims to consider multiple actuator faults on switched systems and to ensure a pole placement of the closed-loop system eigenvalues.

The objective of Fault Tolerant Control system (FTC) is to maintain current performances closed to desirable performances and preserve stability conditions in the presence of component and/or instrument faults. Accommodation capability of a control system depends on many factors such as the severity of the failure, the robustness of the nominal system, and the actuators redundancy. Various approaches for FTC have been suggested in the literature (Noura et al., 2000), (Blanke et al., 2003) and (Zhang and Jiang, 2003) but usually deal with linear systems.

A FTC system is characterized by an on-line and a real-time FDI process and control reconfiguration mechanism. There are two types of control reconfiguration strategies, one is real-time control 
redesign (Wu et al., 2000), (Theilliol et al., 2002), (Rodrigues, 2005), and the other is to switch among pre-designed multiple controllers (Zhang and Jiang, 2001), (Zhang et al., 2005). However, controllers switching underline the fact that many faulty system representations had to be identify so as to synthesize off-line pre-computed and stabilized controllers. These identifications are sometimes difficult to obtain. Moreover, the most of research works in switched systems do not deal with actuator faults, so we develop an output feedback synthesis which can ensure closed-loop system stability despite of faults. This paper extends some previous results in polytopic framework with only state feedback synthesis (Rodrigues et al., 2005) or in LPV case (Rodrigues et al., 2006) under multiple actuator faults.

The paper is organized as follows. The section II gives the problem statement of Fault Tolerant Control in switched systems. In section III, we design a controller synthesis for each actuator and we generate an output feedback control law for switched systems both in fault-free and faulty cases. An illustrative example is given in section IV to underline the FTC method. Finally, concluding remarks are given in the last section. In this paper, the following notations are used: $I_{n}$ denotes an identity matrix of dimension $n \times n$. The dimensions are sometimes omitted in which cases they can be implied from the context.

\section{PROBLEM STATEMENT}

\subsection{Switched systems representation in faulty-case}

Let consider the following switched system with multiple actuator faults (Rodrigues, 2005):

$$
\begin{aligned}
x_{k+1} & =\sum_{j=1}^{N} \alpha_{k}^{j}\left[A_{j} x_{k}+B_{j}\left(I_{p}-\gamma\right) u_{k}\right] \\
y_{k} & =\sum_{j=1}^{N} \alpha_{k}^{j}\left[C_{j} x_{k}\right]
\end{aligned}
$$

where $x \in \mathbb{R}^{n}$ represents the state vector, $u \in$ $\mathbb{R}^{p}$ is the input vector, $y \in \mathbb{R}^{m}$ is the output vector. $\gamma \triangleq \operatorname{diag}\left[\gamma_{1}, \gamma_{2}, \ldots, \gamma_{p}\right], \gamma_{i} \in \mathbb{R}$, such that $\gamma_{i}=1$ represents a total lost, a failure of i-th actuator, $i \in[1, \ldots, p]$ and $\gamma_{i}=0$ implies that i-th actuator operates normally. $A_{j} \in \mathbb{R}^{n \times n}$, $B_{j} \in \mathbb{R}^{n \times p}, C_{j} \in \mathbb{R}^{m \times n}$ are invariant matrices defined for the $j^{\text {th }}$ mode with $j \in[1, \ldots, N]$.

The switched system is scheduled through switching functions designed as follows: $\alpha_{k}^{j} \forall j \in$ $[1, \ldots, N]$ lie in a convex set $\Omega=\left\{\alpha_{k}^{j} \in\right.$ $\mathbb{R}^{N}, \alpha_{k}=\left[\begin{array}{lll}\alpha_{k}^{1} & \ldots \alpha_{k}^{N}\end{array}\right]^{T}, \quad \alpha_{k}^{j} \geq 0 \quad \forall j \quad$ and $\left.\sum_{j=1}^{N} \alpha_{k}^{j}=1\right\}$. These switching functions are supposed to be available in real time depending on parameters measurement and not corrupted by faults. The switching functions $\alpha_{k}^{j}$ are defined as follows:

$$
\alpha_{k}^{j}=\left\{\begin{array}{l}
1 \text { when the switched system is described } \\
\text { by the j-th mode such that: } \\
A(\alpha)=\sum_{j=1}^{N} \alpha_{k}^{j} A_{j}=A_{j} \\
0 \quad \text { otherwise }
\end{array}\right.
$$

So, if $\alpha_{k}^{j}=1$ the system is described by the $j^{\text {th }}$ mode with $\left[A_{j}, B_{j}, C_{j}\right]$.

We propose to develop an Output Feedback for switched systems with actuator failures. Consider the matrix $b_{j}^{i}$ representing total faults in all actuators except the i-th:

$$
B_{j}^{i}=\left[0, \ldots, 0, b_{j}^{i}, 0, \ldots, 0\right]
$$

and $B_{j}=\left[b_{j}^{1}, b_{j}^{2}, \ldots, b_{j}^{p}\right.$, with $b_{j}^{i} \in \mathbb{R}^{n \times 1}$. It is assumed that each column of $B_{j}$ is full column rank whatever the model $j$. As (Maki et al., 2001), the following assumption is considered.

Assumption 1: The pairs $\left(A_{j}, b_{j}^{i}\right), \forall i=1, \ldots, p$ are assumed to be controllable $\forall j=1, \ldots, N$.

\subsection{Principles of Fault Tolerant Control Strategy}

Without loss of generality, it is assumed that when actuator fault occurs on the system, the matrix $\gamma$ can be decomposed as:

$$
\gamma=\left(\begin{array}{cc}
\gamma_{p-h} & 0 \\
0 & I_{h}
\end{array}\right)
$$

with $\gamma_{p-h}$ a diagonal matrix where its elements $\gamma_{p-h}^{i}, i \in[1, \ldots, p]$ are different from 1 which represent the number of actuators not out of order $\left(\gamma^{i} \neq 1\right)$, and $I_{h}$ represents the number $h$ of actuators totally failed. Let define $\Gamma \triangleq\left(I_{p}-\right.$ $\gamma)\left(I_{p}-\gamma\right)^{+}$which represents only totally failed actuators and the corresponding matrix partitions of $B$ such as:

$$
\begin{gathered}
B=\left[\begin{array}{ll}
B_{p-h} & B_{h}
\end{array}\right] \\
B_{p-h} \in \mathbb{R}^{n \times(p-h)} \text { and } B_{h} \in \mathbb{R}^{n \times h} \text { and } \Gamma: \\
\Gamma=\left(\begin{array}{cc}
I_{p-h} & 0 \\
0 & 0_{h}
\end{array}\right)
\end{gathered}
$$

We will present a control law which is able to vanish actuator faults into the state space representation (1) and to ensure closed-loop stability despite of multiple actuator failures. Based on a multiplicative fault representation defined in (1), the new control law $u_{F T C}$ must vanish all actuator faults on the system such that:

$$
u_{F T C}=[I-\gamma]^{+} u_{\text {nom }}
$$

Let introduce the set of indexes of all actuators that are not out of order, i.e.

$$
\Phi \triangleq\left\{i: i \in(1, \ldots, p), \gamma^{i} \neq 1\right\}
$$


and note that $u_{F T C}=[I-\gamma]^{+} u_{\text {nom }}=-[I-$ $\gamma]^{+} F y_{k}=-F_{F T C} y_{k}$ where $F$ is a nominal controller gain and $F_{F T C}$ the new controller gain. So, this specific control law in the state space representation (1) leads to:

$$
\begin{aligned}
B_{j}(I-\gamma) u_{F T C} & =B_{j}(I-\gamma)(I-\gamma)^{+} u_{\text {nom }} \\
& =B_{j} \Gamma u_{\text {nom }}=\sum_{i \in \Phi} B_{j}^{i} u_{\text {nom }}^{i}
\end{aligned}
$$

which avoids actuator fault effect and where $\sum_{i \in \Phi} B_{j}^{i}$ represents the actuators not out of order, i.e. $\sum_{i \in \Phi} B_{j}^{i}=B_{p-h}$ and $u_{n o m}^{i}$ the i-th element of $u_{n o m}$. Due to the fact that each pair $\left(A_{j}, b_{j}^{i}\right), \forall i=$ $1, \ldots, p$ are assumed to be controllable for all $\forall j=$ $1, \ldots, N$, the system still remains controllable despite of actuator failures.

\section{FAULT TOLERANT CONTROL FOR SWITCHED SYSTEMS}

\subsection{Control law synthesis in fault-free case}

Let us recall the multiplicative actuator fault representation on a polytopic system as follows:

$$
\begin{aligned}
x_{k+1} & =\sum_{j=1}^{N} \alpha_{k}^{j}\left[A_{j} x_{k}+\sum_{i=1}^{p} B_{j}^{i}\left(I_{p}-\gamma\right) u_{k}\right] \\
y_{k} & =C x_{k}
\end{aligned}
$$

where $\alpha_{k}^{j}$ represents the switching functions.

Assumption 2: The matrix $C=C_{j}, \forall j \in[1 \ldots N]$ is full row rank.

Assumption 3: Any actuator can fail, but at least one actuator is not lost, which means that the situation $\gamma^{1}=\cdots=\gamma^{p}=1$ is excluded.

In the nominal case, the linear output feedback can be expressed such as:

$$
u_{k}=-F y_{k}
$$

with $y_{k}=C x_{k}$ and $F \in \mathbb{R}^{p \times m}$ is the output feedback controller. In the nominal case $(\gamma=0)$, the representation (10) with a controller $u_{k}=$ $-F y_{k}$ is rewritten as:

$$
\begin{aligned}
x_{k+1} & =\sum_{j=1}^{N} \alpha_{k}^{j}\left[A_{j} x_{k}+B_{j}(I-\gamma)\left(-F y_{k}\right)\right] \\
& =\sum_{j=1}^{N} \alpha_{k}^{j}\left(A_{j}-B_{j} F C\right) x_{k}
\end{aligned}
$$

with the output feedback controller $F$ to determine so as to vanish actuator faults on the system. We want to establish the stability of the closedloop system with a $\mathcal{L} \mathcal{M I}$ pole placement. In order to achieve some desired transient performance, a pole placement should be considered. For many problems, exact pole assignment may not be necessary, it suffices to locate the pole of the closed loop system in a sub-region of the complex left half plane (Chilali and Gahinet, 1996) and (Rodrigues et al., 2005).
So, let define a disk region $\mathcal{L} \mathcal{M I} \mathcal{D}$ included in the unit circle. The pole placement of the closedloop system (12) for all the models $j \in[1 \ldots N]$ in a $\mathcal{L} \mathcal{M I}$ region, can be expressed as the following (Chilali and Gahinet, 1996):

$$
\left(\begin{array}{cc}
-r X & q X+\left(A_{j} X-B_{j} F C X\right)^{T} \\
q X+\left(A_{j} X-B_{j} F C X\right) & -r X
\end{array}\right)<0
$$

However these inequalities are no longer linear with regard to the unknown matrices $X=$ $X^{T}>0$ and $F, \forall j \in[1 \ldots N]$. So, the solution is not guaranteed to belong to a convex domain and the classical tools for solving sets of matrix inequalities cannot be used. It constitutes the major difficulty of output feedback design.

We propose to transform $\mathcal{B M I}$ conditions (13) in $X$ and $F, \forall j \in[1 \ldots N]$, in $\mathcal{L} \mathcal{M I}$ conditions which will be used to synthesize directly a stabilized output feedback. Controllers gains $F_{i}$ are synthesized for each actuator in order to define an output feedback even if there are failures in the system.

Theorem 1. Consider the system (10) in fault-free case $\left(\gamma_{k}=0\right)$, defined $\forall j \in[1 \ldots N]$. Let assume that for each $j$ each pairs $\left(A_{j}, b_{j}^{i}\right)$ are controllable and suppose it is possible to find matrices $X_{i}=$ $X_{i}^{T}>0, M$ and $V_{i} \forall i=[1, \ldots, p]$ such that $\forall i=[1, \ldots, p], \forall j=[1, \ldots, N]$ :

$$
\left(\begin{array}{cc}
-r X_{i} & q X_{i}+\left(A_{j} X_{i}-B_{j}^{i} V_{i} C\right)^{T} \\
q X_{i}+A_{j} X_{i}-B_{j}^{i} V_{i} C & -r X_{i}
\end{array}\right)<0
$$

with

$$
C X_{i}=M_{i} C, \quad \forall i=[1, \ldots, p], \forall j=[1, \ldots, N]
$$

The control law with output feedback $u_{k}=-F y_{k}$ allows to place the eigenvalues of the closed-loop system (10) in a predetermined $\mathcal{L} \mathcal{M I}$-region with $F M=V, F=\sum_{i=1}^{p} G_{i} V_{i}\left(C C^{T}\left(C \sum_{i=1}^{p} X_{i} C^{T}\right)^{-1}\right)$ or $F=V C C^{T}\left(C X C^{T}\right)^{-1}$, with $G_{i}=B_{j}^{i+} B_{j}^{i}, \forall i=$ $[1, \ldots, p], \forall j=[1, \ldots, N]$.

Proof: This proof is similar to (Rodrigues et al., 2005). Summation of (14) for the actuators set $i \in[1, \ldots, p]$ of the system $(10) i=1, \ldots, p$ gives for one model $j, \forall j=[1, \ldots, N]$ :

$$
\sum_{i=1}^{p}\left(\begin{array}{cc}
-r X_{i} & q X_{i}+\left(A_{j} X_{i}-B_{j}^{i} V_{i} C\right)^{T} \\
q X_{i}+A_{j} X_{i}-B_{j}^{i} V_{i} C & -r X_{i}
\end{array}\right)<0
$$

Let denote $X=\sum_{i=1}^{p} X_{i}\left(\right.$ with $\left.X=X^{T}>0\right)$ to obtain

$$
\left(\begin{array}{cc}
-r X & q X+\left(A_{j} X-\sum_{i=1}^{p} B_{j}^{i} V_{i} C\right)^{T} \\
q X+\left(A_{j} X-\sum_{i=1}^{p} B_{j}^{i} V_{i} C\right) & -r X
\end{array}\right)<0
$$


$\forall i=[1, \ldots, p], \forall j=[1, \ldots, N]$. Now, denote the l-th row of the matrix $V_{i}$ as $V_{i}^{l}, i=1, \ldots, p$ and $l=1, \ldots, p$, and $G_{i}=B_{j}^{i+} B_{j}^{i}$ is a matrix equals to zero except in entry $(i, i)$ where there is a one:

$$
V_{i}^{l}=G_{l} V_{i}
$$

Therefore,

$$
\begin{aligned}
& \sum_{i=1}^{p} B_{j}^{i} V_{i} C=\sum_{i=1}^{p}\left[0, \ldots, 0, b_{j}^{i}, 0, \ldots, 0\right] V_{i}^{i} C \\
& =B_{j} \sum_{i=1}^{p} V_{i}^{i} C
\end{aligned}
$$

leading to

$$
\sum_{i=1}^{p} B_{j}^{i} V_{i} C=B_{j} \sum_{i=1}^{p} V_{i}^{i} C=B_{j}\left(\sum_{i=1}^{p} G_{i} V_{i} C\right)
$$

By taking $V=\sum_{i=1}^{p} G_{i} V_{i}$, equation (20) becomes

$$
\sum_{i=1}^{p} B_{j}^{i} V_{i} C=\sum_{i=1}^{p} B_{j}^{i} V_{i} C=B_{j} V C
$$

we get $\forall i=[1, \ldots, p], \forall j=[1, \ldots, N]$

$$
\left(\begin{array}{cc}
-r X & q X+\left(A_{j} X-B_{j} V C\right)^{T} \\
q X+\left(A_{j} X-B_{j} V C\right)-r X
\end{array}\right)<0
$$

With the changes of variables $V=F M$ and $C X=M C$ which substituted in $\mathcal{L} \mathcal{M I}(22)$, leads to

$$
\left(\begin{array}{cc}
-r X & q X+\left(A_{j} X-B_{j} F C X\right)^{T} \\
q X+\left(A_{j} X-B_{j} F C X\right) & -r X
\end{array}\right)<0
$$

$\forall i=[1, \ldots, p], \forall j=[1, \ldots, N]$. These inequalities (23) are $\mathcal{B} \mathcal{M} \mathcal{I}_{\mathrm{s}}$ (13) which could not be solve with classical tools. By multiplying each $\mathcal{L} \mathcal{M I}(22)$ by $\alpha_{k}^{j}$ and summing all of them, we obtain

$$
\left(\begin{array}{cc}
-r X & q X+\sum_{j=1}^{N} \alpha_{k}^{j}\left(A_{j} X-B_{j} V C\right)^{T} \\
q X+\sum_{j=1}^{N} \alpha_{k}^{j}\left(A_{j} X-B_{j} V C\right) & -r X
\end{array}\right)<0
$$

it is equivalent to

$$
\left(\begin{array}{cc}
-r X & q X+(A(\alpha) X-B(\alpha) V C)^{T} \\
q X+(A(\alpha) X-B(\alpha) V C) & -r X
\end{array}\right)<0
$$

with $A(\alpha)=\sum_{j=1}^{N} \alpha_{k}^{j} A_{j}$ and $B(\alpha)=\sum_{j=1}^{N} \alpha_{k}^{j} B_{j}$. Due to the fact that matrix $C$ is supposed to be full row rank, we deduce from (15) there exists a non-singular matrix $M=C X C^{T}\left(C C^{T}\right)^{-1}$ and then after variables changes $F=V M^{-1}=$ $\sum_{i=1}^{p} G_{i} V_{i}\left(C C^{T}\left(C \sum_{i=1}^{p} X_{i} C^{T}\right)^{-1}\right)$. So, quadratic $\mathcal{D}$ stability is ensured by solving (24) with a linear output feedback $u_{k}=-F y_{k}$.

\subsection{Control law synthesis in faulty case}

By considering the system (10) and based on the previous synthesis control law, the FTC method can be developed in this section under assumption that actuator fault estimation $\widehat{\gamma}$ is suitable and known without uncertainty, i.e $\widehat{\gamma}=\gamma$.

Theorem 2. Consider the system (10) in actuator faulty case $\left(\gamma^{i} \neq 0\right)$ under assumption 3 , defined for all modes $j, j=1, \ldots, N$. Let introduce the set of indexes of all actuators that are not completely lost, i.e.

$$
\Phi \triangleq\left\{i: i \in(1, \ldots, p), \gamma^{i} \neq 1\right\}
$$

The Fault Tolerant Control law with a linear output feedback is equivalent to

$$
\begin{aligned}
u_{F T C} & =-(I-\gamma)^{+}\left(\sum_{i \in \Phi} G_{i} V_{i}\left(C C^{T}\left(C \sum_{i \in \Phi} X_{i} C^{T}\right)^{-1}\right)\right) y_{k} \\
& =-(I-\gamma)^{+} F_{r e c} y_{k}=-F_{F T C} y_{k}
\end{aligned}
$$

with $G_{i}=B_{j}^{i+} B_{j}^{i}$, applied to the faulty system (10) allows to constrain pole in prescribed $\mathcal{L} \mathcal{M I}$ region. The output feedback control law $u_{k}=-F_{F T C} y_{k}$ allows to place the eigenvalues of the closed-loop system in predetermined $\mathcal{L} \mathcal{M I}$ region with $F_{\text {rec }} M=V, F_{\text {rec }}=$ $\sum_{i \in \Phi} G_{i} V_{i}\left(C C^{T}\left(C \sum_{i \in \Phi} X_{i} C^{T}\right)^{-1}\right.$ or $F_{r e c}=V C C^{T}\left(C X C^{T}\right)^{-1}$, with $G_{i}=B_{j}^{i+} B_{j}^{i}, \forall i=[1, \ldots, p], \forall j=[1, \ldots, N]$.

Proof:

Applying the new control law (26) to the faulty system (10), leads to the following equation

$B_{j}(I-\gamma) u_{F T C}=-B_{j} \Gamma\left(\sum_{i \in \Phi} G_{i} V_{i}\left(C C^{T}\left(C \sum_{i \in \Phi} X_{i} C^{T}\right)^{-1}\right)\right) y_{k}$

with $\Gamma=(I-\gamma)(I-\gamma)^{+}$defined as

$$
\Gamma=\left(\begin{array}{cc}
I_{p-h} & 0 \\
0 & O_{h}
\end{array}\right)
$$

$\Gamma$ is a diagonal matrix which contains only entries zero (representing total faults) and one (no fault), see section 2.2. Since $B_{j} \Gamma=\sum_{i \in \Phi} B_{i}^{j} \bmod -$ els only the actuators that are not completely lost, then performing the summations in the proof of Theorem (1) over the elements of $\Phi$ shows that $\sum_{i \in \Phi} G_{i} V_{i}\left(C C^{T}\left(C \sum_{i \in \Phi} X_{i} C^{T}\right)^{-1}\right)$ is the output feedback gain matrix for the faulty system $\left(A_{j}, \sum_{i \in \Phi} B_{i}^{j}, C\right)$.

The control law $u_{F T C}$ of the system (26) is realized as:

$$
u_{F T C}=-K_{F T C} y_{k}
$$

with

$$
K_{F T C}=(I-\gamma)^{+} \sum_{i \in \Phi} G_{i} V_{i}\left(C C^{T}\left(C \sum_{i \in \Phi} X_{i} C^{T}\right)^{-1}\right)
$$




\section{ILLUSTRATIVE EXAMPLE}

Let consider the following switched system which consider actuator faults:

$$
\begin{aligned}
x_{k+1} & =\sum_{j=1}^{N} \alpha_{k}^{j} A_{j} x_{k}+\sum_{j=1}^{N} \alpha_{k}^{j} B_{j}\left(I-\gamma_{k}\right) u_{k} \\
y_{k} & =C x_{k}
\end{aligned}
$$

with the set of matrices described as follows:

$$
\begin{gathered}
A_{1}=\left[\begin{array}{cccc}
0.75 & 0 & 0 & 0 \\
0 & 0.85 & 0 & 0 \\
0 & 0 & 0.75 & 0 \\
0 & 0 & 0 & 0.9
\end{array}\right], B_{1}=\left[\begin{array}{cc}
0.95 & 0.95 \\
1 & 1 \\
0.9 & 0.9 \\
1 & 1
\end{array}\right] \\
A_{2}=\left[\begin{array}{cccc}
0.75 & 0 & 0 & 0 \\
0 & 0.85 & 0 & 0 \\
0 & 0 & 0.8 & 0 \\
0 & 0 & 0 & 0.9
\end{array}\right], B_{2}=\left[\begin{array}{cc}
1.05 & 1.05 \\
1 & 1 \\
0.9 & 0.9 \\
1 & 1
\end{array}\right] \\
A_{3}\left[\begin{array}{cccc}
0.75 & 0 & 0 & 0 \\
0 & 0.85 & 0 & 0 \\
0 & 0 & 0.70 & 0 \\
0 & 0 & 0 & 1.1
\end{array}\right], A_{4}=\left[\begin{array}{cccc}
0.75 & 0 & 0 & 0 \\
0 & 0.85 & 0 & 0 \\
0 & 0 & 0.8 & 0 \\
0 & 0 & 0 & 1.1
\end{array}\right] \\
B_{3}=\left[\begin{array}{cc}
0.95 & 0.95 \\
1 & 1 \\
1.1 & 1.1 \\
1 & 1
\end{array}\right], B_{4}=\left[\begin{array}{cc}
1.05 & 1.05 \\
1 & 1 \\
1.1 & 1.1 \\
1 & 1
\end{array}\right], C=\left[\begin{array}{llll}
0 & 1 & 0 & 0 \\
0 & 0 & 1 & 0 \\
0 & 0 & 0 & 1
\end{array}\right]
\end{gathered}
$$

The switched system (31) is described by 4 modes defined previously. The modes of such switched system are represented through matrices $A_{j}, B_{j}, C$ with parameters $\alpha_{k}^{j}=1, \alpha_{k}^{i}=0, i \neq j$. The following matrices are directly linked with Theorems (1) and (2), with parameters $q=-0.05$ and $r=0.93$ :

$$
\begin{gathered}
V_{1}=\left[\begin{array}{ccc}
-0.0863 & -0.0889 & -0.1051 \\
0 & 0 & 0
\end{array}\right] \\
M_{1}=M_{2}\left[\begin{array}{ccc}
0.9951 & -0.0004 & 0.0629 \\
-0.0004 & 1.0000 & 0.0054 \\
0.0629 & 0.0054 & 0.1895
\end{array}\right]
\end{gathered}
$$

and

$$
\begin{gathered}
X_{1}=X_{2}\left[\begin{array}{cccc}
1 & 0 & 0 & 0 \\
0 & 0.9951 & -0.0004 & 0.0629 \\
0 & -0.0004 & 1 & 0.0054 \\
0 & 0.0629 & 0.0054 & 0.1895
\end{array}\right] \\
V_{2}=\left[\begin{array}{cccc}
0 & 0 & 0 \\
-0.0863 & -0.0889 & -0.1051
\end{array}\right]
\end{gathered}
$$

with $F=V M^{-1}=\sum_{i=1}^{p} G_{i} V_{i}\left(C C^{T}\left(C \sum_{i=1}^{p} X_{i} C^{T}\right)^{-1}\right)$ :

$$
F=\left[\begin{array}{lll}
0.0265 & 0.0430 & 0.2674 \\
0.0265 & 0.0430 & 0.2674
\end{array}\right], G_{1}=\left[\begin{array}{ll}
1 & 0 \\
0 & 0
\end{array}\right], G_{2}=\left[\begin{array}{ll}
0 & 0 \\
0 & 1
\end{array}\right]
$$

At the same sample $k=2$, the first actuator is out of order and a fault of $90 \%$ loss of effectiveness appears on the second actuator. The matrix $\gamma$ is equal to

$$
\gamma=\left[\begin{array}{cc}
1 & 0 \\
0 & 0.9
\end{array}\right], k \geq 2
$$

The figure (1) represents respectively in fault-free case: the output vector evolution in (a), state vector evolution in (b), evolution of the second actuator in (c) and the first actuator in (d), and finally parameters evolution $\alpha_{k}^{j}$ in (e). The closedloop system is stable without any fault. The figure (2) represents the same characteristics evolution as in figure (1) with an actuator failure on the 1st actuator and a fault of $90 \%$ in the 2 nd actuator at sample $k=2$. This figure illustrates the instability of the closed-loop system in faulty-case.

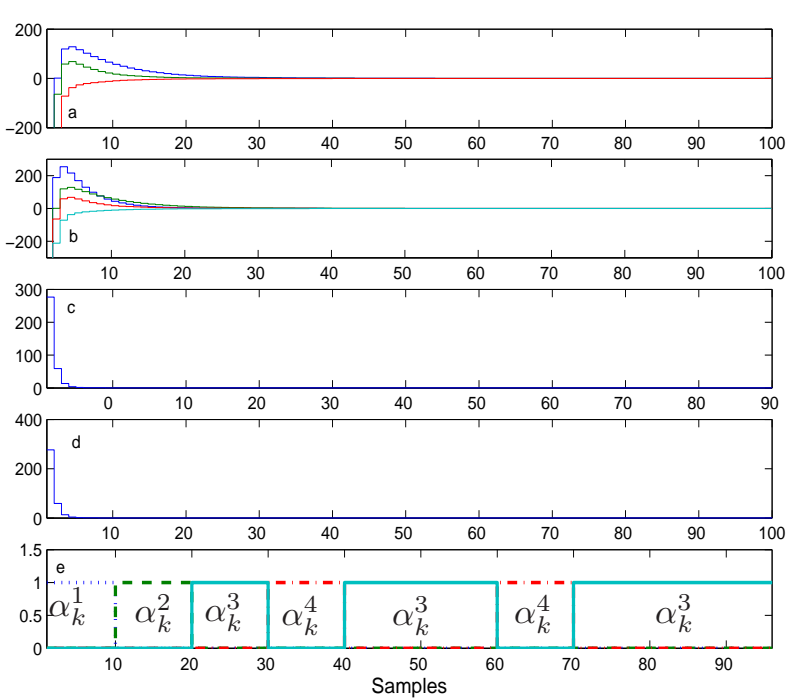

Fig. 1. Nominal case: (a) Evolution of the outputs, (b) Evolution of the states, (c) Evolution of the 2nd actuator, (d) Evolution of the 1st actuator, (e) Evolution of parameters $\alpha_{k}^{j}$
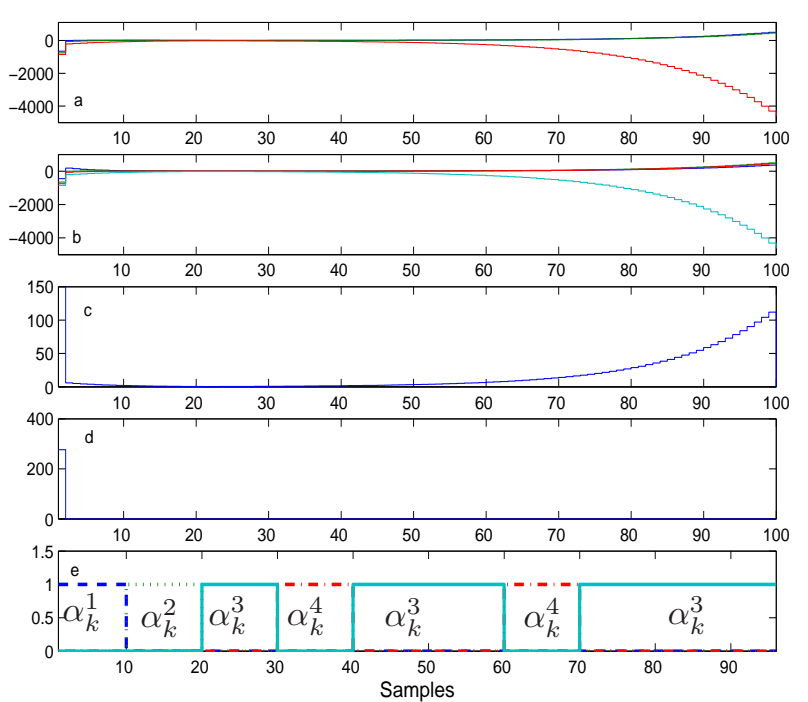

Fig. 2. In faulty case: (a) Evolution of the outputs, (b) Evolution of the states, (c) Evolution of the 2nd actuator, (d) Evolution of the 1st actuator, (e) Evolution of parameters $\alpha_{k}^{j}$

The figure (3) underlines the contribution of Theorems (1) and (2) applied to switched systems. The Fault Tolerant Control law remains the system stable despite of multiple actuator faults. In order to simulate a time delay provided from FDI block, the new control law is only applied at sample $k=15$. (Shin, 2003) discusses issues with a time delay in FTC reconfiguration. The reader could refer to this NASA/NIA Report for more information on time delay in reconfiguration.

\section{CONCLUSION}

The method developed in this paper emphasises the importance of the Fault Tolerant Control for switched systems. A controller is designed for 

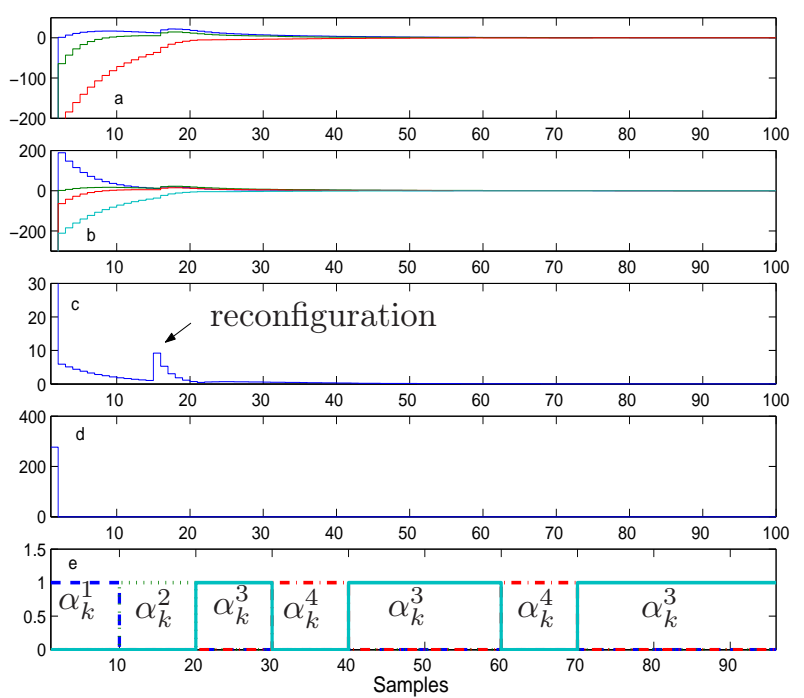

Fig. 3. Reconfiguration: (a) Evolution of the outputs, (b) Evolution of the states, (c) Evolution of the 2nd actuator, (d) Evolution of the 1st actuator, (e) Evolution of parameters $\alpha_{k}^{j}$

each separate actuator through an $\mathcal{L} \mathcal{M} \mathcal{I}$ pole placement in fault-free case and faulty case. It allows the system to continue operating safely, to avoid stopping it immediately and to ensure stability despite the presence of actuator failures. The synthesis of a linear output feedback takes into account the information provided by a FDI scheme. An illustrative example with switched system has been presented to demonstrate the effectiveness of the scheme.

\section{REFERENCES}

Blanke, M., M. Kinnaert, J. Lunze and M. Staroswiecki (2003). Diagnosis and Fault Tolerant Control. Edts Springer-Verlag.

Chadli, M., D. Maquin and J. Ragot (2002). An lmi formulation for output feedback stabilization in multiple model approach. In: Proc. of the 41'st IEEE Conf. on Decision and Control, Las Vegas, USA. pp. 311-316.

Chilali, M. and P. Gahinet (1996). $H_{\infty}$ design with pole placement constraints: an LMI approach. IEEE Trans. on Automatic Control 41(3), 358-367.

Daafouz, J. and J. Bernussou (2002). Robust dynamic output feedback control for switched systems. In: Proc. of the 41'st IEEE Conf. on Decision and Control, Las Vegas, USA. pp. 4389-4394.

Daafouz, J., P. Riedinger and C.Iung (2003). Stabilizing switched control design with performances. In: Proc. IFAC Conference on Analysis and Design of Hybrid Systems, ADHS'03, Saint-Malo, France.

Liberzon, D. and A. Stephen Morse (1999). Basic problems in stability and design of switched systems. IEEE Control Systems 19(5), 59-70.
Maki, M., J. Jiang and K. Hagino (2001). A stability guaranteed active fault-tolerant control system against actuator failures. In: Proc. of the 40th IEEE Conference on Decision and Control, Orlando, Florida.

Mignone, D., G. Ferrari-Trecate and M. Morari (2000). Stability and stabilization of piecewise affine and hybrid systems: An lmi approach. In: Proc. of the 39'th IEEE Conf. on Decision and Control,Sydney, Australia.

Noura, H., D. Sauter, F. Hamelin and D. Theilliol (2000). Fault-tolerant control in dynamic systems: Application to a winding machine. IEEE Control Systems Magazine pp. 33-49.

Rodrigues, M. (2005). Diagnostic et commande active tolérante aux défauts appliqués aux systèmes décrits par des multi-modèles linéaires. Phd thesis. Centre de Recherche en Automatique de Nancy, UHP. Nancy, France.

Rodrigues, M., D. Theilliol and D. Sauter (2005). Design of an Active Fault Tolerant Control and Polytopic Unknown Input Observer for Systems described by a Multi-Model Representation. In: Proc. 44th IEEE Conference on Decision and Control and European Control Conference ECC, Sevilla, Spain.

Rodrigues, M., D. Theilliol and D. Sauter (2006). Active Actuator Fault Tolerant Control Design for Polytopic LPV Systems. In: Proc. Proc. 6th IFAC Symposium Safeprocess, Beijing, China.

Shin, J-Y. (2003). Parameter transient behavior analysis on fault tolerant control system. Technical Report NASA-CR-2003-212682NIA Report No. 2003-05. National Institute of Aerospace. Hampton, Virginia, USA.

Theilliol, D., H. Noura and J.C. Ponsart (2002). Fault diagnosis and accommodation of threetank system bsaed on analytical redundancy. ISA Transactions 41, 365-382.

Wu, N. E., Y. Zhang and K. Zhou. (2000). Detection, estimation and accommodation of loss of control effectiveness. Int. J. of Adaptive Control and Signal Processing 14(7), 775-795.

Zhang, Y. and J. Jiang (2001). Integrated active Fault-Tolerant Control using IMM approach. IEEE Transactions on Aerospace and Electronics Systems 37(4), 1221-1235.

Zhang, Y. and J. Jiang (2003). Bibliographical review on reconfigurable Fault-Tolerant Control systems. In: Proc. IFAC Symposium Safeprocess, Washington. D.C, USA, CD-Rom.

Zhang, Y., J. Jiang, Z. Yang and A. Hussain (2005). Managing performance degradation in Fault Tolerant Control Systems. In: Proc. 16th IFAC World Congress, Prague, Czech Republic. 